\title{
Closed loop supply chain mathematical modeling considering lean agile resilient and green strategies
}

\author{
Masoud Mohammadzadeh ${ }^{1}$, Mohammadali Sobhanallahi ${ }^{1, *}$ and Alireza Arshadi \\ Khamseh $^{1}$ \\ 1 Department of Industrial Engineering, Faculty of Engineering \\ Kharazmi University, No. 43 South Mofatteh Ave., Tehran, Iran \\ E-mail: 〈mohammadzadeh.masoud@yahoo.com〉,〈sobhanallahi@khu.ac.ir $\rangle$, \\ 〈alireza.arshadikhamseh@gmail.com〉
}

\begin{abstract}
The supply chain management is planning, implementation and effective control of supply chain operations considered as a key factor for the competitiveness of the organizations. To make these targets, four management strategies of lean, agile, resilient and green have been separately proposed. Recently, studies have been performed with a consideration of these four strategies simultaneously named LARG (Lean, Agile, Resilient and Green). However, due to the novelty of this subject, the mathematical modeling of SCND (Supply Chain Network Design) has not been addressed in LARG strategy. SCND is one of the most essential parts of supply chain management that strategic decisions of it have heavily effects in both overall and partial applicability of the supply chain. The goal of this paper is to design a closed loop supply chain network considering LARG strategy using multi-objective modeling with uncertain demand. The objective functions are total profit, customer satisfaction and total pollution. The model is formulated to determine which facility sites should be selected (strategic decisions), and find out the optimal number of parts and products in the network (tactical decisions). Finally, a real industrial case study is provided to illustrate the performance and applicability of the LARG strategy in SCND in practice.
\end{abstract}

Keywords: LARG strategy, mathematical modeling, supply chain network design, uncertain demand

Received: February 18, 2020; accepted: October 15, 2020; available online: December 18, 2020

DOI: $10.17535 /$ crorr.2020.0015

\section{Introduction}

Lean, agile, resilient and green strategies have been introduced and used in supply chain management [2]. The lean strategy aims to reduce operational costs. To this end, it possibly conflicts with the green strategy, which aims to reduce ecological impact on the industry activities thus potentially adding operational costs and inflexible operations. Further, green firms focus on long-term strategic supplier alliances and integrating their work into one or fewer suppliers, resulting in a smaller supply base. Conversely, resilient supply chains count on alternative sourcing and alternative transportation to respond to unexpected negative events. This reduces the promptitude with which customer needs can be addressed. This objective also possibly quarrels with the agile strategy, which aims to respond quicker to customer needs.

Literature review shows that most of the researches have only studied one of the strategies or the combination of two or three strategies up to now [12]. Considering four different strategies in supply chain management synchronicity, is quite important, but it is a challenging issue due to the fact that each of these strategies has different methods and ways to improve the performance

*Corresponding author. 
of the chain. As a strategy, the lean strategy is based on cost reduction and flexibility, and is focused on eliminating and identifying the various sources of waste [2]. The agile strategy refers to flexible and timely actions in response to the rapid change environments [15]. The green strategy focuses primarily on consolidating environmental awareness into management practices [18]. The resilient strategy considers the effects of external factors on the supply chain and focuses on sustaining the supply chain during critical situations [6].

In other example, lean desires to minimize the level of inventory to almost zero, since it is highly dependent on Just-In-Time (JIT) inventory management. The agile and resilient strategies suggest adequate safety stock levels to respond effectively to the change in demands. In fact, the capacity surplus lets the supply chain manager to answer the unpredictable happenings in costumer's demands yet it doesn't mean that the supply chain must have a very high capacity surplus as it is unacceptable in terms of cost and function [5].

The agile strategy reckons on mass customization as a manufacturing planning in order to respond to market requirements. Conversely, the resilience strategy punctuates minimal batch sizes and capacity redundancies to respond to market uncertainty in a similar way to the agile strategy. The agile strategy, however, takes advantage of changes, while the resilience strategy focuses only on the negative events of the business environment that lead failure. Additionally, the lean strategy endeavors to maintain a high average utilization rate by using the pull system as a manufacturing planning. The green strategy focuses on manufacturing capabilities to effectively combine reusable/remanufactured components.

These four strategies, besides having negative effects on some methods, are compatible and positively effective on the other methods [4]. For instance, increasing the integration level of the chain, reducing the production delays, transportation time, increasing the informing frequency in the chain are the common methods of the mentioned strategies. Therefore, in addition to the necessity of having all four strategies for supply chain management, the managers need to achieve a balance between the available methods and strategies. Simultaneously considering all these four strategies has led to a new strategy known as LARG strategy. The LARG strategy can be considered as models of thinking within modern management strategies in the supply chain context that aims to address and manage the entire supply chain members and urges to achieve sustainable businesses and a competitive advantage.

It is obvious that each strategy considers a different aspect of the supply chain, while using LARG supply chain management approach can combine the methods of all the mentioned strategies simultaneously and it can be used to improve the effectiveness, function and competition in supply chain management to survive in dynamic and diverse markets [17]. Carvalho et al. [5] provided a conceptual model for stating the differences and similarities of these four management strategies to reach a more competitive and sustainable supply chain. They have introduced three main criteria for evaluation: service level, preparation time and cost. At the end, they showed the effects of each management strategy on these criteria. Azevedo et al. [2] have also suggested a conceptual model to improve the operational, economic and environmental function of supply chains according to methods and activities of lean, agile, resilient and green strategies. They suggested examination of the LARG combination approach as a suitable strategy to improve the function of supply chain experimentally. Maleki and Cruz Machado [12] introduced an approach for integration of lean, agile, resilient, and green strategies according to the values of the customer in car industry via analyzing busy networks. They categorized the guidance of LARG strategy according to the methods of production, assembling, and logistic and then generalized it to six customers' values (quality, cost, environment consideration, knowledge, customization and time). The results of this research are important for the supply chain members.

Cabral et al. [4] used key decision making method of Analytical Network Process (ANP) to prioritize the function of the supply chain and lean, agile, resilient and green strategies according to four key indicators of function: service level, cost, time and quality. This research 
has studied the supply chain of Volkswagen but because of using network analysis process method, the number of clusters are too much. Cabrita et al. [15] studied combining LARG and BMC (Business Model Concave) principles. The literature on this subject shows that there is a deep gap between these two scopes. This research helps the discussion of creating an ideal business model compatible with LARG strategy.

Suifan et al. [16] propose an integrated multi-criteria decision method to analyze trade-offs among lean, agile, resilient and green paradigms in supply chain management associated with competitive priorities. They employ the entropy method to derive the alternative weights of the evaluation criteria, and then use TOSIS technique to rank feasible alternatives in order of preference, and finally measure trade-offs among the conflicting objectives. They identify the trade-offs in the Jordan pharmaceutical supply chains by an empirical study approach.

Amjad et al. [1] study the possibility of collaboration between elements of LARG manufacturing with aspects of industry 4.0 . They discovered that LARG elements can be combined with various aspects of industry 4.0 to provide operational, environmental and economic benefits.

To summarize, the focus on LARG scope researches has been on conceptual models and evaluation for choosing the best strategy and lack of modeling and network design in this area is sensible. One of the perfect models of closed loop supply chain network design has been suggested by Mohammadzadeh et al. [14]. They suggested a two-step model for closed loop supply chain in which demand is uncertain. In the first step, they discussed on evaluation and ranking suppliers and in the next step the closed loop supply chain is expanded.

The remainder of this paper is organized as follows. In Section 2, methodology and mathematical modeling is presented. In Section 3 the proposed model is tested and validated in a real-life case study. Finally, conclusions and future studies are drawn in Section 4.

\section{Methodology and mathematical modeling}

\subsection{Discussion}

Studying the literature indicated that some methods of the four strategies are common, such as reduction of preparation time and increased shared information, and do not interfere with each other. Since these have no contradictions in the four strategies, their introduction would only increase the model size of LARG supply chain design without adding any new theoretical value. However, some conflicting points were found in the literature review of the subject.

Capacity surplus: the capacity surplus is effective for reducing the risk in both productive and storehouse parts while they reduce being lean and green and increase the agile and resilient features of the chain [13].

Scattered facility: the lean strategy tries to reduce the number and dispersion of facilities to decrease the surplus cost. Conversely, the agile strategy requires more facilities so that there is more flexibility, and they are closer to the customers. The green strategy wants lower facilities so that the pollution emitted is minimal knowing that two facilities with specific capacity have more pollution compared to a single facility with a total capacity of the two. Consequently, the resilient strategy requires having many scattered facilities such that if an accident happens in a particular location, many of the facilities do not get involved and the supply chain is preserved [8].

Transportation vehicle: there is a limited number of different transportation vehicles accessible for the overall supply chain. Vehicles with different pollution, cost and capacity which effects reloading and the size of the packages. Lean strategy insists on using the cheapest vehicle. Notwithstanding, the green strategy requires selecting the vehicle with the least pollution. The agile strategy requires using low capacity vehicles to increase the reloading rate and faster response to the customer. Moreover, the resilience strategy agrees to benefit a variety of transportation vehicles in order to reduce transportation disruption risks [11]. 
In this research, a closed loop supply chain will be modeled according to these three critical points. The effects of three challenging point of four management strategies summarized in Table 1.

\begin{tabular}{|lcccc|}
\hline \multirow{2}{*}{ Challenging point } & Strategy & & & \\
\cline { 2 - 5 } & Lean & Agile & Green & Resilient \\
\hline \hline Capacity surplus & $\downarrow$ & $\uparrow$ & $\uparrow$ & $\downarrow$ \\
ScatteredfFacility & $\downarrow$ & $\uparrow$ & $\uparrow$ & $\downarrow$ \\
Transportation vehicle & least cost & least capacity & various vehicle & least pollution \\
\hline
\end{tabular}

Table 1: Challenging points effects on strategies

\subsection{Model hypothesis}

As depicted in Figure 1, the network consists of different markets, disassembly centers, refurbishing centers, part inventories, producers, distributers and suppliers. The network has a multi-production flow of different parts with predetermined likelihood of presence. Market points, retailers and collecting centers are the same. So, retail centers in all the market collect the returned products as well. The produce's needs will be supplied simultaneously by external suppliers and part inventories. Demand has normal distribution function; however, the type of distribution function is not effective in the proposed model, and it is used only in the process of normalization. The suppliers' capacities are unlimited. Transferring lines are unbounded. There are several vehicles for transportation with different cost, capacity and pollution. Destruction centers will be provided by the government. The notations are categorized by sets and indices, parameters and decision variables and presented in Appendix A.

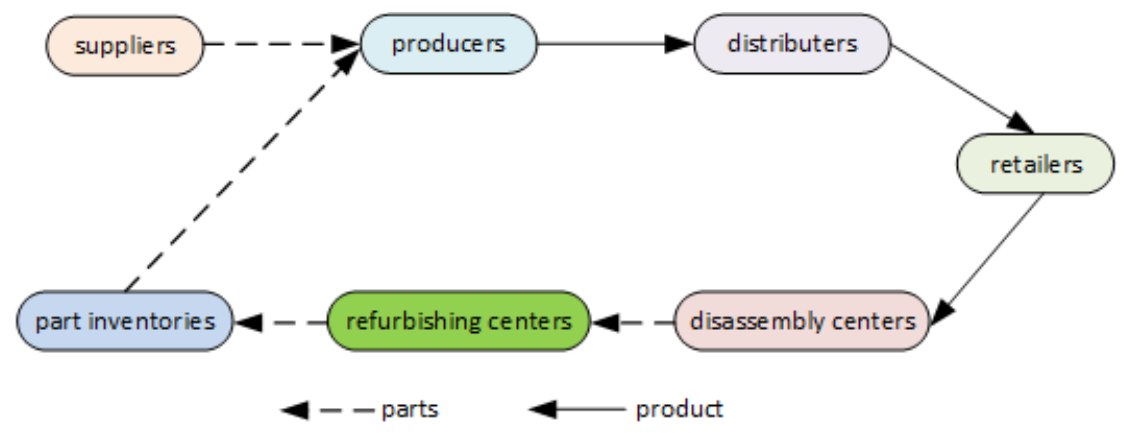

Figure 1: Structure of the proposed closed-loop supply

\subsection{Multi-objective model}

Now the multi-objective mathematical model of the hypothesis will be provided.

Objective functions

For modeling, three objective functions that were used more often in the literature for supply chain have been considered: 1) overall profit that must be maximized, 2) emitted pollution that must be minimized, and 3) customer satisfaction that must be maximized.

First the profit of selling product $j$ in retail $c$ must be calculated. To this end, the following signs are introduced as $(x \wedge D)=\min \{x, D\}, a^{+}=\max \{0, a\}$.

Profit of selling product $j$ in retail $c$ is stated as (1). Its first part shows the selling income which is equal to the price of one unit of the product $j$ sold in retail c multiplies by all the sold products in retail $c$. the next part indicates the unsold products' value and the third part 
conveys the shortage cost which is equal to amount of shortage multiple price of each shortage unit.

$$
s_{j c}\left(x_{j c} \wedge D_{j c}\right)+v s_{j c}\left(x_{j c}-D_{j c}\right)^{+}-u s_{j c}\left(D_{j c}-x_{j c}\right)^{+} .
$$

This paper uses newspaper boy problem in order to calculate inventory control system in retailers in single period. Accordingly, the supply chain manager estimates the sales amount in next period and orders it at the right time before the beginning of the next period. The unsold product and shortage amount create surplus value and shortage cost. This method considers demand uncertainty. The above equation equals to equation (2) as explained in Appendix B. Because $E\left(D_{j c}\right) u s_{j c}$ is constant, it is not effective in the value of decision variables and will be eliminated.

$$
\sum_{j, c}\left(s_{j c}+u s_{j c}\right) x_{j c}-E\left(D_{j c}\right) u s_{j c}+\left(v s_{j c}-s_{j c}-u s_{j c}\right) \int_{0}^{x_{j c}} F\left(D_{j c}\right) d D_{j c} .
$$

This part formulizes the existing costs in this closed loop supply chain. Buying cost: this equals to total costs for buying parts from external suppliers.

$$
\sum_{i, k, m, t} s p r_{i k} g_{i k m t} .
$$

Placement cost: equation (4) shows total placement cost of each facility which is allocated.

$$
\sum_{m} f m_{m} x m_{m}+\sum_{d} f d_{d} x d_{d}+\sum_{s} f s_{s} x s_{s}+\sum_{r} f r_{r} x r_{r}+\sum_{p} f p_{p} x p_{p} .
$$

Current cost of sending path: this cost is illustrated in equation (5). The first term indicates the total sending costs of parts from part inventories to producers. The second term displays the total sending cost of parts from suppliers to producers. Third term is total operational costs in producers. Fourth term implies the total production sending costs from producers to distributers. Fifth term is total operational costs in distributers. Sixth term is total cost of the sending products from distributers to retailers, finally seventh term represents total operational costs in retailers.

$$
\begin{aligned}
& \sum_{i, p, m, t} t n_{p m t} n_{i p m t}+\sum_{i, k, m, t} t g_{k m t} g_{i k m t}+\sum_{j, m} o_{j m} o m_{j m}+\sum_{j, m, d, t} t f_{m d t} f_{j m d t}+ \\
& \sum_{j, d}\left(o d_{j d} \sum_{m, t} f_{j m d t}\right)+\sum_{j, d, c, t} t e_{d c t} e_{j d c t}+\sum_{j, c}\left(o c_{j c} \sum_{d, t} e_{j d c t}\right) .
\end{aligned}
$$

Current cost of returning path: the equation (6) implies this cost. First term indicates total cost of sending products from retailers to disassembly centers, second term conveys total costs of operation in disassembly centers, third term displays the total costs of sending from disassembly centers to refurbishing centers, fourth term implies total operational costs in refurbishing centers, fifth term represents total sending costs from refurbishing centers to part inventories, finally the sixth term express total operational costs in storehouses.

$$
\begin{aligned}
& \sum_{j, c, s, t} t h_{c s t} h_{j c s t}+\sum_{j, s}\left(o s_{j s} \sum_{j}\left(q_{j i} \sum_{c, t} h_{j c s t}\right)\right)+\sum_{j, s, r, t} t l_{s r t} l_{i s r t}+ \\
& \sum_{i, r}\left(o r_{i r} \sum_{s, t} l_{i s r t}\right)+\sum_{i, r, p, t} t v_{r p t} v_{i r p t}+\sum_{i, p}\left(o p_{i p} \sum_{r, t} v_{i r p t}\right) .
\end{aligned}
$$


Opportunity cost: Construction of facilities brings costs and while the whole capacity of the facility is not used, it is called a missed opportunity. The cost of this opportunity can be considered as a percentage of unused capacity multiplies by the cost of facility opportunity. The equation (7) represents that opportunity cost equals to the total opportunity cost of all the potential points of the facilities.

$$
\begin{aligned}
& \sum_{m}\left(1-a m_{m}\right) q m_{m} x m_{m}+\sum_{d}\left(1-a d_{d}\right) q d_{d} x d_{d}+\sum_{s}\left(1-a s_{s}\right) q s_{s} x s_{s}+ \\
& \sum_{r}\left(1-a r_{r}\right) q r_{r} x r_{r}+\sum_{p}\left(1-a p_{p}\right) q p_{p} x p_{p} .
\end{aligned}
$$

Therefore, total profit of the activity of this supply chain will be:

$$
\begin{aligned}
\max z_{1}= & \sum_{j, c}\left(s_{j c}+u s_{j c}\right) x_{j c}+\left(v s_{j c}-s_{j c}-u s_{j c}\right) \int_{0}^{x_{j c}} F\left(D_{j c}\right) d D_{j c} \\
- & \left(\sum_{i, k, m, t} s p r_{i k} g_{i k m t}+\sum_{m} f m_{m} x m_{m}+\sum_{d} f d_{d} x d_{d}+\sum_{s} f s_{s} x s_{s}+\sum_{r} f r_{r} x r_{r}+\right. \\
& \sum_{p} f p_{p} x p_{p}+\sum_{i, p, m, t} t n_{p m t} n_{i p m t}+\sum_{i, k, m, t} t g_{k m t} g_{i k m t}+\sum_{j, m} o_{j m} o m_{j m}+\sum_{j, m, d, t} t f_{m d t} f_{j m d t}+ \\
& \sum_{j, d}\left(o d_{j d} \sum_{m, t} f_{j m d t}\right)+\sum_{j, d, c, t} t e_{d c t} e_{j d c t}+\sum_{j, c}\left(o c_{j c} \sum_{d, t} e_{j d c t}\right)+\sum_{j, c, s, t} t h_{c s t} h_{j c s t}+ \\
& \sum_{j, s}\left(o s_{j s} \sum_{j}\left(q_{j i} \sum_{c, t} h_{j c s t}\right)\right)+\sum_{j, s, r, t} t l_{s r t} l_{i s r t}+\sum_{i, r}\left(o r_{i r} \sum_{s, t} l_{i s r t}\right)+ \\
& \sum_{i, r, p, t} t v_{r p t} v_{i r p t}+\sum_{i, p}\left(o p_{i p} \sum_{r, t} v_{i r p t}\right)+\sum_{m}\left(1-a m_{m}\right) q m_{m} x m_{m}+\sum_{d}\left(1-a d_{d}\right) q d_{d} x d_{d}+ \\
& \left.\sum_{s}\left(1-a s_{s}\right) q s_{s} x s_{s}+\sum_{r}\left(1-a r_{r}\right) q r_{r} x r_{r}+\sum_{p}\left(1-a p_{p}\right) q p_{p} x p_{p}\right) .
\end{aligned}
$$

Pollution: the calculation of the total amount of pollution (Carbon Dioxide) for transferring between facilities has been conducted. In this calculation several vehicles with different levels of contamination and effective distances on pollution have been considered. Then the pollution amount of unused capacity will be considered as surplus capacity of the contaminant. Due to the amount of pollution for placing each facility, total pollution equals to sum of pollution amount if the facility is placed in the potential location. Total produced pollution is as follows:

$$
\begin{aligned}
\min z_{2}= & \sum_{i, m, k, t} p g_{k m t} g_{i k m t}+\sum_{j, m, d, t} p f_{m d t} f_{j m d t}+\sum_{j, d, c, t} p e_{d c t} e_{j d c t}+\sum_{j, c, s, t} p h_{c s t} h_{j c s t}+ \\
& \sum_{i, r, s, t} p l_{s r t} l_{i s r t}+\sum_{i, r, p, t} p v_{r p t} v_{i r p t}+\sum_{i, p, m, t} p m_{p m t} n_{i p m t}+\sum_{m}\left(1-a m_{m}\right) e m_{m}+ \\
& \sum_{d}\left(1-a d_{d}\right) e d_{d}+\sum_{s}\left(1-a s_{s}\right) e s_{s}+\sum_{r}\left(1-a r_{r}\right) e r_{r}+\sum_{p}\left(1-a p_{p}\right) e p_{p}+ \\
& \sum_{m} p m_{m} x m_{m}+\sum_{d} p d_{d} x d_{d}+\sum_{s} p s_{s} x s_{s}+\sum_{r} p r_{r} x r_{r}+\sum_{p} p p_{p} x p_{p}
\end{aligned}
$$


Customer's satisfaction: This research refers to a customer not just as the final consumer but each facility is considered as a customer of the previous facility in the supply chain. The first term defines customer's satisfaction while using maximum capacity of the facilities. It is calculated by summing of multiplication of used facility percentage in the amount of satisfaction of facility using. Moreover, the second term is the amount of satisfaction from dispersion and number of facilities which is defined as the number of created facilities divided to total provided facilities multiples by the amount of satisfaction from variety of facilities. And finally, the third term is the quantity of satisfaction for fast delivery. The capacity of vehicles is in contradiction to the pace of it, so the function is determined by division of total displacement between two facilities on capacity of vehicle multiples by satisfaction level between two facilities.

$$
\begin{aligned}
& \max z_{3}=\sum_{m} a m_{m} \times s m_{m}+\sum_{d} a d_{d} \times s d_{d}+\sum_{s} a s_{s} \times s s_{s}+\sum_{r} a r_{r} \times s r_{r}+\sum_{p} a p_{p} \times s p_{p} \\
& +d m \frac{\sum_{m} x m_{m}}{M}+d d \frac{\sum_{d} x d_{d}}{D}+d s \frac{\sum_{s} x s_{s}}{S}+d r \frac{\sum_{r} x r_{r}}{R}+d p \frac{\sum_{p} x p_{p}}{P} \\
& +\sum_{m, d} s f_{m d}\left(\sum_{j, t} \frac{f_{j m d t}}{c f_{m d t}}\right)+\sum_{d, c} s e_{d c}\left(\sum_{j, t} \frac{e_{j d c t}}{c e_{d c t}}\right)+\sum_{c, s} s h_{c s}\left(\sum_{j, t} \frac{h_{j c s t}}{c h_{c s t}}\right) \\
& +\sum_{s, r} s l_{s r}\left(\sum_{i, t} \frac{l_{i s r t}}{c l_{s r t}}\right)+\sum_{r, p} s v_{r p}\left(\sum_{i, t} \frac{v_{i r p t}}{c v_{r p t}}\right)+\sum_{p, m} s n_{p m}\left(\sum_{i, t} \frac{n_{i p m t}}{c n_{p m t}}\right)+\sum_{k, m} s g_{k m}\left(\sum_{i, t} \frac{g_{i k m t}}{c g_{k m t}}\right)
\end{aligned}
$$

\section{Constraints}

Possibility limitation: equations (11)-(21) show that if facilities placed in suggested locations, there will be a likelihood of sending and receiving products and parts.

$$
\begin{array}{ll}
\sum_{j, t, d} f_{j m d t} \leq M \times x m_{m} & \forall m \\
\sum_{i, p, t} n_{i p m t} \leq M \times x m_{m} & \forall m \\
\sum_{i, k, t} g_{i k m t} \leq M \times x m_{m} & \forall m \\
\sum_{c, t, j} e_{j d c t} \leq M \times x d_{d} & \forall d \\
\sum_{j, t, m} f_{j m d t} \leq M \times x d_{d} & \forall d \\
\sum_{i, r, t} l_{i s r t} \leq M \times x s_{s} & \forall s \\
\sum_{j, c, t} h_{j c s t} \leq M \times x s_{s} & \forall s \\
\sum_{i, p, t} v_{i r p t} \leq M \times x r_{r} & \forall r \\
\sum_{i, s, t} l_{i s r t} \leq M \times x r_{r} & \forall r
\end{array}
$$




$$
\begin{array}{ll}
\sum_{i, m, t} n_{\text {ipmt }} \leq M \times x p_{p} & \forall p \\
\sum_{i, r, t} v_{i p r t} \leq M \times x p_{p} & \forall p
\end{array}
$$

Limitations of network equilibrium: equation (22) indicates that the number of entered parts to producer equals to the number of requested parts. Equation (23) enforces producers to send all products to distributers. Equation (24) implies that the number of entered products to distributers equal to the number of exited ones. Equation (25) conveys that the number of sent products to retailer equals to the total sent products from all the distributers. Equation (26) ensures that the number of sent products to disassembly centers equals to the returned number. Equation (27) indicates that the number of parts provided in each disassembly center equals to the number of returned products multiples by the number of existing parts in each product. Equation (28) represents that the number of sent parts to refurbishing center equals to healthy parts provided in disassembly center. Equation (29) ensures that the number of entered parts into refurbishing center equals the exited parts from the same center. Equation (30) shows that balance level in the inventory parts.

$$
\begin{array}{cc}
\sum_{j} q_{i j} o_{j m}=\sum_{k, t} g_{i k m t}+\sum_{p, t} n_{i p m t} \quad \forall m, i \\
o_{j m}=\sum_{d, t} f_{j m d t} \quad \forall m, j \\
\sum_{m, t} f_{j m d t}=\sum_{c, t} e_{j d c t} \quad \forall d, j \\
x_{j c}=\sum_{d, t} e_{j d c t} \quad \forall c, j \\
y_{j} \times x_{j c}=\sum_{s, t} h_{j c s t} \quad \forall s, j \\
b_{i s}=\sum_{c, j, t} h_{j c s t} q_{i j} & \forall s, i \\
\left(1-w_{i}\right) b_{i s}=\sum_{r, t} l_{i s r t} & \forall i, s \\
\sum_{p, t} v_{i r p t}=\sum_{s, t} l_{\text {isrt }} & \forall i, r \\
\sum_{m, t} n_{i p m t}=\sum_{r, t} v_{i r p t} & \forall p, i
\end{array}
$$

Capacity limitations: equation (31) states that if producer $\mathrm{m}$ places in suggested location, its total product must be less or equal to the capacity of this producer. Equation (32) shows that if distributer d places in suggested location, total distributed product cannot be more than its capacity. Equation (33) implies that if disassembly center s places in suggested location, all the disassembly part is restricted to the capacity of this center. Equation (34) implies that if refurbishing center $\mathrm{r}$ is placed in suggested location, total refurbishing operation is limited to its capacity. Equation (35) ensures that if part inventory $\mathrm{p}$ is located in suggested location, total part that can be stored in the place is bounded to its capacity. 


$$
\begin{array}{cc}
\sum_{j} o_{j m} \leq\left(c m_{m} \times x m_{m} \times a m_{m}\right) & \forall m \\
\sum_{c, t, j} e_{j d c t} \leq\left(c d_{d} \times x d_{d} \times a d_{d}\right) & \forall d \\
\sum_{i} b_{i s} \leq\left(c s_{s} \times x s_{s} \times a s_{s}\right) & \forall s \\
\sum_{i, s, t} e_{i s r t} \leq\left(c r_{r} \times x r_{r} \times a r_{r}\right) & \forall r \\
\sum_{r, t, i} v_{i r p t} \leq\left(c p_{p} \times x p_{p} \times a p_{p}\right) & \forall p
\end{array}
$$

Possibility of capacity limitation: equations (36)-(40) state that if facilities are located in a certain potential location, the capacity of that facility can be used.

$$
\begin{array}{cc}
a m_{m} \leq x m_{m} & \forall m \\
a d_{d} \leq x d_{d} & \forall d \\
a s_{s} \leq x s_{s} & \forall s \\
a r_{r} \leq x r_{r} & \forall r \\
a p_{p} \leq x p_{p} & \forall p
\end{array}
$$

Decision variables: constraints (41) and (42) enforce the binary and non-negativity restrictions on the decision variables.

$$
\begin{gathered}
g_{i k m t}, f_{j m d t}, e_{j d c t}, h_{j c s t}, l_{i s r t}, v_{i r p t}, n_{i p m t}, o_{j m}, b_{i s}, x_{j c}, a m_{m}, a d_{d}, a s_{s}, a r_{r}, a p_{p} \geq 0 \\
x m_{m}, x d_{d}, x s_{s}, x r_{r}, x p_{p}, x k_{k} \in\{0,1\}
\end{gathered}
$$

\subsection{Linearization method}

The presented model is nonlinear due to the integral in the objective function. This study uses piecewise linearization used in some researches including [3]. The range of linear function must be divided into a number of intervals, and the curve must be replaced with straight lines which are recognized with a constant and a gradient. For standard normal distribution which is common among demand distribution of retailers, the estimation can be seen in the Figure 2. This estimation converts nonlinear function into linear one and helps us reach the optimal answer of linearized function. The accuracy of this estimation method will be increased by raising the quantity of divisions and intervals and each interval must have an index and a zero-one variable. 


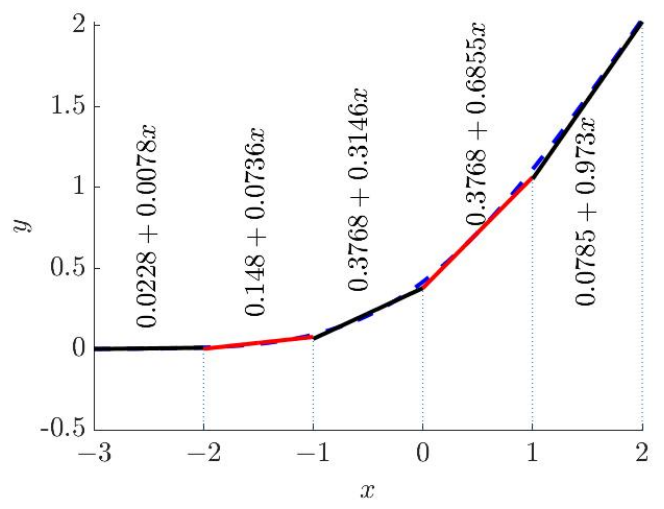

Figure 2: Linear estimation of integral of normal collective distribution function

Indexes

$n_{c}^{j} \in N_{c}^{j}$ : sets of approximation intervals for each product in every retailer

\section{Parameters}

coef $f_{n_{c}^{j}}:$ interval gradient of $n_{c}^{j}$

const $_{n_{c}^{j}}$ : constant of interval $n_{c}^{j}$

lower $_{n_{c}^{j}}$ : lower limit of interval $n_{c}^{j}$

upper $_{n_{c}^{j}}$ : upper limit of interval $n_{c}^{j}$

Decision variables

bint $_{n_{c}^{j}}$ : is one if interval $n_{c}^{j}$ is chosen

aint $t_{c}^{j}$ : sent product if interval $n_{c}^{j}$ is chosen

After introducing the above notations, the linear model is as follows:

$$
\begin{aligned}
\max z_{1} & =\sum_{j, c}\left(\left(s_{j c}+u s_{j c}\right) \sum_{n_{c}^{j}} a i n t_{n_{c}^{j}}+\left(v s_{j c}-s_{j c}-u s_{j c}\right) \sum_{n_{c}^{j}}\left(\operatorname{coeff}_{n_{c}^{j}} \times \text { aint }_{n_{c}^{j}}+\text { const }_{n_{c}^{j}} \times \text { bint }_{n_{c}^{j}}\right)\right) \\
& -\left(\sum_{i, k, m, t} s p r_{i k} g_{i k m t}+\sum_{m} f m_{m} x m_{m}+\sum_{d} f d_{d} x d_{d}+\sum_{s} f s_{s} x s_{s}+\sum_{r} f r_{r} x r_{r}+\right. \\
& \sum_{p} f p_{p} x p_{p}+\sum_{i, p, m, t} t n_{p m t} n_{i p m t}+\sum_{i, k, m, t} t g_{k m t} g_{i k m t}+\sum_{j, m} o_{j m} o m_{j m}+\sum_{j, m, d, t} t f_{m d t} f_{j m d t}+ \\
& \sum_{j, d}\left(o d_{j d} \sum_{m, t} f_{j m d t}\right)+\sum_{j, d, c, t} t e_{d c t} e_{j d c t}+\sum_{j, c}\left(o c_{j c} \sum_{d, t} e_{j d c t}\right)+\sum_{j, c, s, t} t h_{c s t} h_{j c s t}+ \\
& \sum_{j, s}\left(o s_{j s} \sum_{j}\left(q_{j i} \sum_{c, t} h_{j c s t}\right)\right)+\sum_{j, s, r, t} t l_{s r t} l_{i s r t}+\sum_{i, r}\left(o r_{i r} \sum_{s, t} l_{i s r t}\right)+ \\
& \sum_{i, r, p, t} t v_{r p t} v_{i r p t}+\sum_{i, p}\left(o p_{i p} \sum_{r, t} v_{i r p t}\right)+\sum_{m}\left(1-a m_{m}\right) q m_{m} x m_{m}+\sum_{d}\left(1-a d_{d}\right) q d_{d} x d_{d}+ \\
& \left.\sum_{s}\left(1-a s_{s}\right) q s_{s} x s_{s}+\sum_{r}\left(1-a r_{r}\right) q r_{r} x r_{r}+\sum_{p}\left(1-a p_{p}\right) q p_{p} x p_{p}\right) \\
\min z_{2} & =\ldots \\
\max z_{3} & =\ldots
\end{aligned}
$$


s.t.

$(41)-(49)$

$$
\begin{gathered}
\sum_{n_{c}^{j}} \text { bint }_{n_{c}^{j}}=1 \\
\text { aint }_{n_{c}^{j}} \leq \operatorname{upper}_{n_{c}^{j}} \times \operatorname{bint}_{n_{c}^{j}} \quad \forall n_{c}^{j} \\
\text { aint }_{n_{c}^{j}} \geq \operatorname{lower}_{n_{c}^{j}} \times \operatorname{bint}_{n_{c}^{j}} \quad \forall n_{c}^{j} \\
\operatorname{bint}_{n_{c}^{j}} \in\{0,1\} \\
\operatorname{aint}_{n_{c}^{j}} \geq 0 \\
\sum_{n_{c}^{j}} \text { aint }_{n_{c}^{j}}=\sum_{d, t} e_{j d c t} \quad \forall c, j \\
\sum_{n_{c}^{j}} y_{j} \times \operatorname{aint}_{n_{c}^{j}}=\sum_{s, t} h_{j c s t} \quad \forall c, j
\end{gathered}
$$

Equation (43) states that only one of the intervals must be chosen for each product for every retailer. Equations (44)-(45) represent that the quantity of delivery products must be between the lower and upper limits of the interval. Equation (46) states binary variables. Equation (47) implies the positivity of the flow amount through the network. Equation (48) indicates that the summation of products in interval equals to total deploying quantities to retailers and equation (49) ensures whole quantity of parts in the intervals are equal to total sent flow to disassembly centers.

In the first objective function and the limitations (31)-(35) there is a nonlinear statement which consists of multiplying a continuous variable and a zero-one variable. Therefore, in order to linearize it, some new binary and continuous variables with additional constraints are introduced as reported in Table 2. Using Glover and Woolsey [7] method which is as follows, limitations can be linearized.

$$
\begin{aligned}
& z=x_{1} \times x_{2} \quad x_{1}=0 \text { or } 1 \quad 0 \leq x_{2} \leq 1 \\
& \equiv \\
& z \leq x_{2} \quad z \leq M \times x_{1} \quad z \geq x_{2}-M \times\left(1-x_{1}\right) \quad z \geq 0
\end{aligned}
$$

And in objective function, the nonlinear equation will be linearized as follows.

$$
\begin{array}{r}
\max z_{1}=(\ldots)-\left(\ldots+\sum_{m}\left(x m_{m}-a x m_{m}\right) q m_{m}+\sum_{d}\left(x d_{d}-a x d_{d}\right) q d_{d}\right. \\
\left.+\sum_{s}\left(x s_{s}-a x s_{s}\right) q s_{s}+\sum_{r}\left(x r_{r}-a x r_{r}\right) q r_{r}+\sum_{p}\left(x p_{p}-a x p_{p}\right) q p_{p}\right)
\end{array}
$$

\subsection{Multi-objective solution method}

For solving the considered multi-objective model, Huang and Yun [9] approach will be used in which the aim is finding a solution near to the ideal amount being resulted by minimizing the following function. In this equation $\mathrm{y}$ is the number of objective function and $\mathrm{p}$ is a number mostly between 1 and 2. The weights are calculated via FAHP method. Refer to [10] in order 


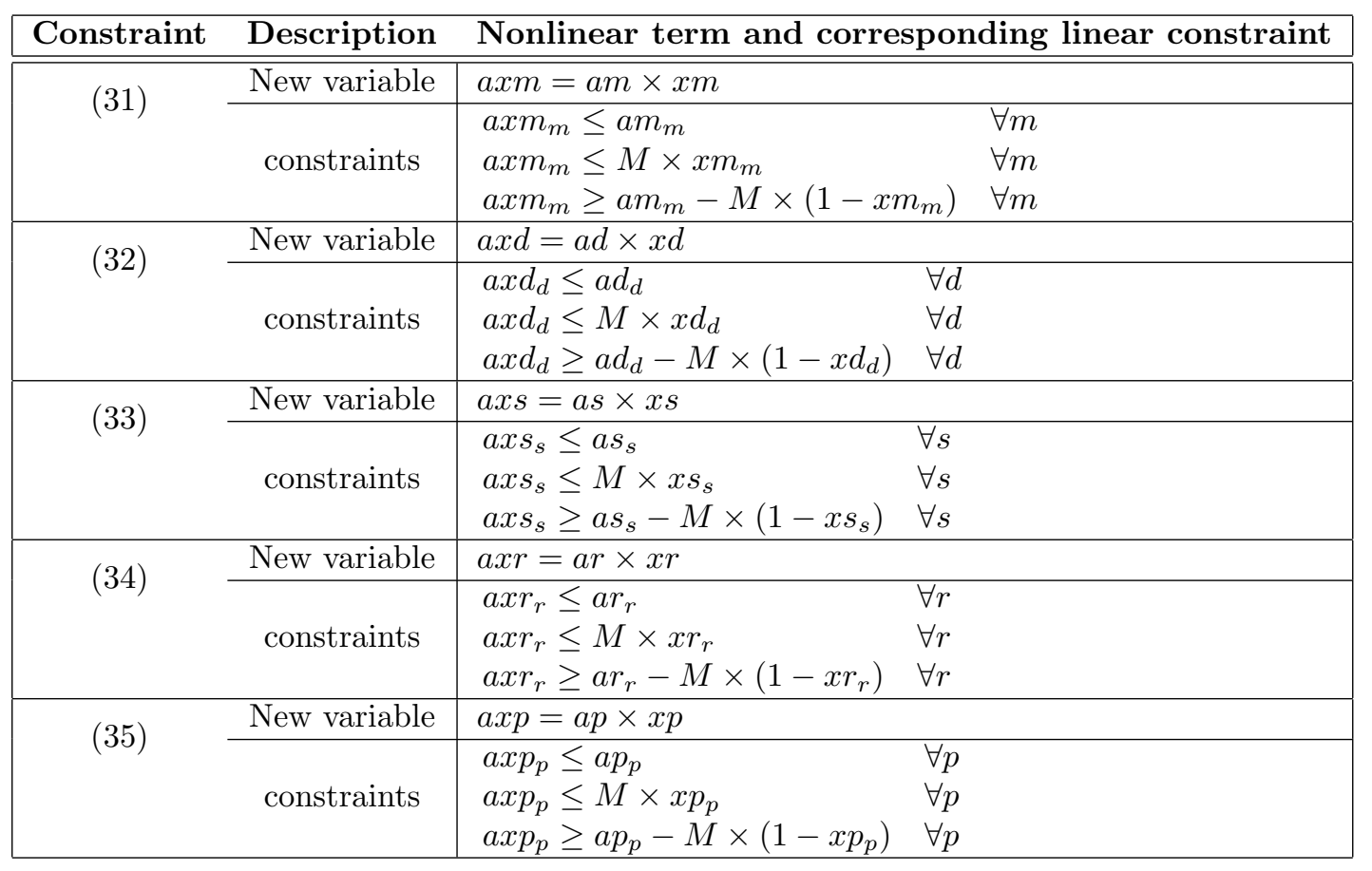

Table 2: Categories of variables change and linearization

to study more on this subject. Z+ and Z- are the best and the worst amount of each objective functions in single-objective solution.

$$
l_{p}=\left[\sum_{y=1}^{Y} w_{y}^{p}\left(\frac{z_{y}-z_{y}^{-}}{z_{y}^{+}-z_{y}^{-}}\right)^{p}\right]^{1 / p}
$$

According to these explanations, linear single-objective model combined with constant of the problem is calculated as follows:

$$
\max z=\left[w_{1}^{p}\left(\frac{z_{1}-z_{1}^{-}}{z_{1}^{+}-z_{1}^{-}}\right)^{p}+w_{2}^{p}\left(\frac{z_{2}-z_{2}^{-}}{z_{2}^{+}-z_{2}^{-}}\right)^{p}+w_{3}^{p}\left(\frac{z_{3}-z_{3}^{-}}{z_{3}^{+}-z_{3}^{-}}\right)^{p}\right]^{1 / p}
$$

After linearization and providing a method of solving multi-objective the problem can solved via optimization software.

\section{Results and discussion}

\subsection{A real-life case study}

In this section, some information is informed about the company where we research case study on. Iran School Equipment Company was established in 1977 with the aim of designing and producing various types of classroom equipment and furniture. Enjoying 50000 square meter work and production space in a 60000 square meter plot of land in addition to a modern R\&D department and highly experienced experts, this company is capable enough to develop its products according to the latest worldwide standards and modern technologies. The market for classroom equipment in Iran is about 1000 billion rials per year, and the company has a large part of this market. The company produces a variety of products that in this study we have 
focused on the following three products: Desk and bench, Student chair and Teacher's desk that each of them is composed of different parts. These products are intended for sale in three target markets that each of them has a retailer who also has the responsibility of collecting returned products. The related information is as follow.

Specifications of all markets including selling price of products, salvage value of unsold products, shortage cost of lack of products, and demands are shown in the Table 3. Return rate for all the products is $10 \%$ and the percentage of non-usable for all the parts is considered as 15\%. There are four types of vehicles for transportation among facilities: Truck; with petrol or hybrid motor and Trailer; with petrol or hybrid motor. Table 4 shows the capacity, cost and pollution of each four types of vehicles between all facilities. In this research only the $\mathrm{CO} 2$ emitted in the proposed supply chain network has been considered $(\mathrm{CO} 2 \mathrm{Kg} / \mathrm{Km})$. It should be noted that applications of this kind of supply chain network models to the real cases are really hard. As it can be seen, some parameters are random numbers that choose from uniform distribution.

\begin{tabular}{|l|cccc|}
\hline & $\left(S_{j c}\right)$ & $\left(s v_{j c}\right)$ & $\left(s c_{j c}\right)$ & $\left(D_{c j}\right)$ \\
\hline \hline J1.c1 & 200 & 148 & 50 & 1040 \\
$\mathrm{~J} 2 . c 1$ & 175 & 110 & 60 & 2300 \\
$\mathrm{~J} 3 . c 1$ & 150 & 100 & 50 & 1960 \\
$\mathrm{~J} 1 . c 2$ & 112 & 91 & 70 & 7970 \\
$\mathrm{~J} 2 . c 2$ & 530 & 60 & 50 & 1600 \\
$\mathrm{~J} 3 . c 2$ & 150 & 100 & 50 & 1500 \\
$\mathrm{~J} 1 . c 3$ & 175 & 90 & 60 & 2200 \\
$\mathrm{~J} 2 . c 3$ & 150 & 100 & 60 & 8060 \\
$\mathrm{~J} 3 . c 3$ & 200 & 158 & 60 & 2350 \\
\hline
\end{tabular}

Table 3: Product related parameters in markets

\begin{tabular}{|l|llll|}
\hline & $t 1$ & $t 2$ & $t 3$ & $t 4$ \\
\hline \hline cost & $\mathrm{u}(13,17)$ & $\mathrm{u}(9,10)$ & $\mathrm{u}(6,7)$ & $\mathrm{u}(4,5)$ \\
capacity & $\mathrm{u}(8,9)$ & $\mathrm{u}(10,12)$ & $\mathrm{u}(19,21)$ & $\mathrm{u}(23,25)$ \\
pollution & $\mathrm{u}(6,8)$ & $\mathrm{u}(9,11)$ & $\mathrm{u}(29,35)$ & $\mathrm{u}(42,50)$ \\
\hline
\end{tabular}

Table 4: Parameters of transportation vehicle

Satisfaction from fast transportation of parts and products between facilities is considered as a random number between 50 and 90 from uniform distribution. In this research, we consider the time as indicator of customers satisfaction. Decision making group state the importance of each objective function as $\mathrm{w} 1=0.5, \mathrm{w} 2=0.2, \mathrm{w} 3=0.3$ and $\mathrm{p}=1$. In order to linearize the first objective function, five intervals have been considered. The GAMS software has been used for this problem solving.

The result of solving model is as follows: profit is 1102489 and total pollution is 570742 and customer satisfaction is 25219. The first producer manufactures the second and third products while second one produces only first product and third producer have all the products in production plan. First refurbishing center refurbishes the first and the second parts while the second refurbishing center refurbishes only the second and the third and fourth parts and third refurbishing center recycling only the first part. 


\subsection{The benefit of the proposed model}

This section aims to investigate how the LARG mathematical modeling can assist in improving the objective functions. To this end, this paper compares the objective functions of the following two approaches for the supply chain network design.

LARG mathematical modeling: This approach makes the SCND decisions using the LARG mathematical modeling presented in the previous section.

Sequential approach: This approach, first solves the model without considering one of the four strategies and then by replacement of decision variables (transportation amounts, construct locations and used capacity percentages) in objective functions, calculate the objective functions values.

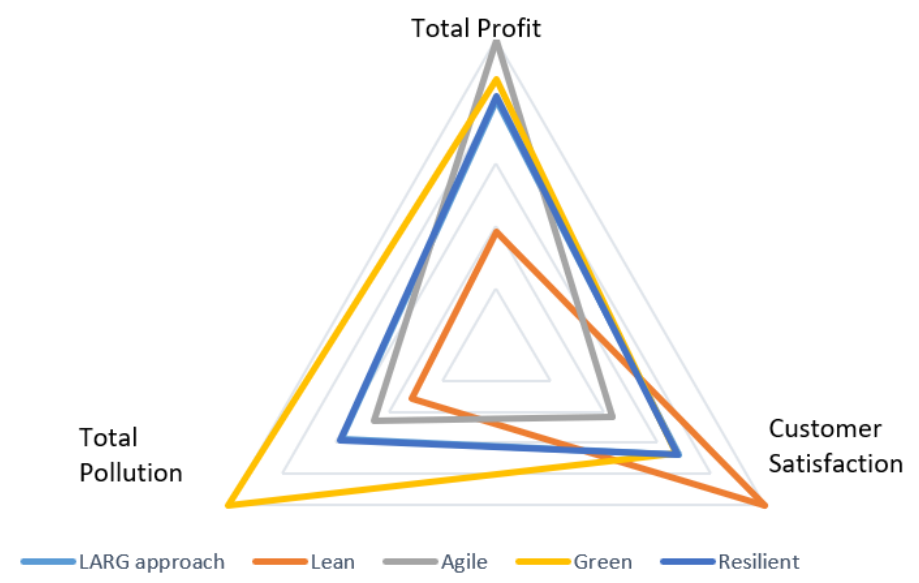

Figure 3: Comparison bitween LARG approach and sequential approach

For example, by carelessly lean strategy in 3 changeling points, the mathematical model earns the value of all decision variables. And then with replacing this decision variables in profit objective function, the value of it is calculated. This approach repeated for agile and green strategies in turn. The amount of three objective functions in the sequential approach is showed in Figure 3.

Differences between the sequential approach and LARG approach also are provided in Table 5. The last row of Table 5 presents the change percentages weighted by the weights of objective functions. It is obvious from the last row that by eliminating each strategy, the weighted objective function decline.

This numerical example proofs the efficiency of LARG mathematical modeling in SCND. Also, the preference of LARG strategy again the separately four strategy has been established.

Elimination resilient strategy haven't specific effect on weighted objective function because of action of resilient strategy is in the implementation stage of the supply chain but we must consider resilient strategy in design stage to avoid of difficulties in implementation stage.

\begin{tabular}{|llllll|}
\hline \multirow{2}{*}{ Objective Function } & \multirow{2}{*}{ LARG Approach } & \multicolumn{4}{l|}{ Eliminated Strategy } \\
\cline { 3 - 6 } & & Lean & Agile & Green & Resilient \\
\hline \hline Total profit (w1=0.5) & 1120489 & 531172 & 1389941 & 1213171 & 1137749 \\
Total pollution (w2=0) & 570742 & 313548 & 450190 & 991542 & 57729 \\
Customer satisfaction (w3=0) & 25219 & 37112 & 15991 & 24891 & 24970 \\
Weighted change percentage & & $-3.1 \%$ & $-2.6 \%$ & $-3.1 \%$ & $00.2 \%$ \\
\hline
\end{tabular}

Table 5: Comparing the performance of the sequential and LARG approaches 


\section{Conclusion and future direction}

The conscience of industrial engineering and particularly, the conscience of the supply chain management is unification and that is the motivation of the LARG supply chain management. Necessarily, all the organizations would move towards LARG supply chain management which has more advantages in theoretical objective functions compared to each individual strategy and being more successful in the current multi-aspect nature of the supply chains. On the other hand, design of the supply chain network effects the long-term performance of the supply chain and disregarding the main strategies of the supply chain management during the design period, leads to incompatibility during the operation phase of the supply chain. Having a large diversion between these two fields is one of the theoretical shortcomings of the supply chain management.

As mentioned in this paper in detail, four strategies; lean, agile, resilient and green have been considered separately or sometimes researchers jointed both of these in their works. There are several conflicts here, such as capacity surplus, scattered facility and transportation vehicle. Here a mathematical model has been developed which combined all these conflicts in a model with three objective functions as: profit, pollution and customer satisfaction. By linearization of nonlinear equations and using compromise programming method, this problem has been solved.

We illustrated the model output for a real-life case study from the classroom equipment industry that showed significant improvements in the objective function due to considering all four strategies simultaneously. This paper demonstrates LARG capabilities in CSND. Proves that the LARG ability in CSND is very high and it is very useful in theory and practice. In practice, it helps the executive manager to consider all management methods simultaneously that causes the manager does not worry about losing any resources. In theory, it indicates that the optimal solution obtained through LARG is better than the optimal solution obtained from solving the model without considering any of the strategies.

For future studies, more conflict issues would be considered as challenging point of the integrated model for LARG strategies. Entrance of inventory control and routing into model would be another investigation topic. Considering facilities disruption can also provide additional insights and practical implications.

\section{References}

[1] Amjad, M. S., Rafique, M. Z., Hussain, S., and Khan, M. A. (2020). A new vision of LARG manufacturing - A trail towards industry 4.0. CIRP Journal of Manufacturing Science and Technology. doi: 10.1016/j.cirpj.2020.06.012

[2] Azevedo, S. G., Carvalho, H., and Machado, V. C. (2011). The influence of green practices on supply chain performance: A case study approach. Transportation research part E: logistics and transportation review, 47(6), 850-871. doi: 10.1016/j.tre.2011.05.017

[3] Baghalian, A., Rezapour, S., and Farahani, R. Z. (2013). Robust supply chain network design with service level against disruptions and demand uncertainties: A real-life case. European Journal of Operational Research, 227(1), 199-215. doi: 10.1016/j.ejor.2012.12.017

[4] Cabral, I., Grilo, A., and Cruz-Machado, V. (2012). A decision-making model for lean, agile, resilient and green supply chain management. International Journal of Production Research, 50(17), 4830-4845. doi: 10.1080/00207543.2012.657970

[5] Carvalho, H., Duarte, S., and Machado, V. C. (2011). Lean, agile, resilient and green: Divergencies and synergies. International Journal of Lean Six Sigma, 2(2), 151-179. doi: $10.1108 / 20401461111135037$

[6] Espadinha-Cruz, P., Grilo, A., Puga-Leal, R., and Cruz-Machado, V. (2011). A model for evaluating Lean, Agile, Resilient and Green practices interoperability in supply chains. IEEE Interna- 
tional Conference on Industrial Engineering and Engineering Management 2011, 120-1213. doi: 10.1109/IEEM.2011.6118107

[7] Glover, F., and Woolsey, E. (1974). Converting the 0-1 polynomial programming problem to a 0-1 linear program. Operations research, 22(1), 180-182. doi: 10.1287/opre.22.1.180

[8] Hosseini, S., and Barker, K. (2016). A Bayesian network model for resilience-based supplier selection. International Journal of Production Economics, 180, 68-87. doi: 10.1016/j.ijpe.2016.07.007

[9] Hwang, C. L., and Yoon, K. (1981). Multiple attribute decision making - Mmethods and ppplications. New York: Springer-Verlag Berlin Heidelberg. doi: 10.1007/978-3-642-48318-9

[10] Kahraman, C., Cebeci, U., and Ulukan, Z. (2003). Multi-criteria supplier selection using fuzzy AHP. Logistics information management, 16(3), 382-394. doi: 10.1108/09576050310503367

[11] Khalili, S. M., Jolai, F., and Torabi, S. A. (2017). Integrated production-distribution planning in two-echelon systems: A resilience view. International Journal of Production Research, 55(4), 1040-1064. doi: 10.1080/00207543.2016.1213446

[12] Maleki, M., and Cruz-Machado, V. (2013). Generic integration of Lean, Agile, Resilient, and Green practices in automotive supply chain. Review of International Comparative Management/Revista de Management Comparat International, 14(2), 237-248. http://www.rmci.ase.ro/no14vol2/ 05.pdf

[13] Margolis, J. T., Sullivan, K. M., Mason, S. J., and Magagnotti, M. (2018). A multi-objective optimization model for designing resilient supply chain networks. International Journal of Production Economics, 204, 174-185. doi: 10.1016/j.ijpe.2018.06.008

[14] Mohammadzadeh, M., Khamseh, A. A., and Mohammadi, M. (2017). A multi-objective integrated model for closed-loop supply chain configuration and supplier selection considering uncertain demand and different performance levels. Journal of Industrial \& Management Optimization, 13(2), 1041-1064. doi: 10.3934/jimo.2016061

[15] do Rosário Cabrita, M., Duarte, S., Carvalho, H., and Cruz-Machado, V. (2016). Integration of lean, agile, resilient and green paradigms in a business model perspective: Theoretical foundations. IFAC-PapersOnLine, 49(12), 1306-1311. doi: 10.1016/j.ifacol.2016.07.704

[16] Suifan, T., Alazab, M., and Alhyari, S. (2019). Trade-off among lean, agile, resilient and green paradigms: an empirical study on pharmaceutical industry in Jordan using a TOPSISentropy method. International Journal of Advanced Operations Management, 11(1-2), 69-101. doi: 10.1504/IJAOM.2019.10019905

[17] Tizroo, A., Azar, A., Ahmadi, R., and Rafiei, M. (2011). Modeling agility of supply chain case study: Zobahan Co. Journal of Industrial Managemant, 3(7), 17-36. https://www.sid.ir/en/ journal/ViewPaper . aspx?id=415806

[18] Zhu, Q., Sarkis, J., and Lai, K. H. (2008). Confirmation of a measurement model for green supply chain management practices implementation. International Journal of Production Economics, 111(2), 261-273. doi: 10.1016/j.ijpe.2006.11.029

\section{Appendix A}

The notations are categorized by sets and indices, parameters and decision variables.

Sets and indices

$i \in I$ : sets of parts in all the products

$j \in J:$ sets of products

$k \in K$ : sets of potential suppliers

$m \in M$ : sets of potential producers

$d \in D:$ sets of potential distributors

$c \in C:$ sets of retailers (market)

$s \in S:$ sets of potential disassembly centers 
$r \in R:$ sets of potential refurbishing centers

$p \in P$ : sets of potential part inventories

$t \in T:$ series of existing transportation vehicles

\section{Parameters}

$t f_{m d t}$ : sending cost of a product from producer $m$ to distributor $d$ with transportation vehicle $t$

$t e_{d c t}$ : sending cost of a product from distributor $d$ to retailer $c$ with transportation vehicle $t$

$t h_{c s t}$ : sending cost of a product from retailer $c$ to disassembly center $s$ with transportation vehicle $t$

$t l_{s r t}$ : sending cost of a part from disassembly center $s$ to refurbishing center $r$ with transportation vehicle $t$

$t v_{r p t}$ : sending cost of a part from refurbishing center $r$ to part inventory $p$ with transportation vehicle $t$

$t n_{p m t}$ : sending cost of a part from part inventory $p$ to producer $m$ with transportation vehicle $t$

$t g_{k m t}$ : sending cost of a part from supplier $k$ to producer $m$ with transportation vehicle $t$

$p g_{k m t}$ : caused pollution by sending a part from supplier $k$ to producer $m$ with transportation vehicle $t$

$p f_{m d t}$ : caused pollution by sending a product from producer $m$ to distributor $d$ with transportation vehicle $t$

$p e_{d c t}$ : caused pollution by sending a product from distributor $d$ to retailer $c$ with transportation vehicle $t$

$p h_{c s t}$ : caused pollution by sending a product from retailer $c$ to disassembly center $s$ with transportation vehicle $t$

$p l_{s r t}$ : caused pollution by sending a part from disassembly center $s$ to refurbishing center $r$ with transportation vehicle $t$

$p v_{r p t}$ : caused pollution by sending a part from refurbishing center $r$ to part inventory $p$ with transportation vehicle $t$

$p n_{p m t}$ : caused pollution by sending a part from part inventory $p$ to producer $m$ with transportation vehicle $t$

$c f_{m d t}$ : capacity of transportation vehicle $t$ for sending product from producer $m$ to distributor $d$

$c g_{k m t}$ : capacity of transportation vehicle $t$ for sending part from supplier $k$ to producer $m$

$c e_{d c t}$ : capacity of transportation vehicle $t$ for sending product from distributor $d$ to retailer $c$

$c h_{c s t}$ : capacity of transportation vehicle $t$ for sending product from retailer $c$ to disassembly center $s$

$c l_{s r t}$ : capacity of transportation vehicle $t$ for sending part from disassembly center $s$ to refurbishing center $r$ 
$c v_{r p t}$ : capacity of transportation vehicle $t$ for sending part from refurbishing center $r$ to part inventory $p$

$c n_{p m t}$ : capacity of transportation vehicle $t$ for sending part from part inventory $p$ to producer $m$

$s f_{m d}$ : satisfaction of fast delivery of products from producer $m$ to distributor $d$

$s e_{d c}$ : satisfaction of fast delivery of products from distributor $d$ to retailer $c$

$s h_{c s}$ : satisfaction of fast delivery of products from retailer $c$ to disassembly center $s$

$s l_{s r}$ : satisfaction of fast delivery of parts from disassembly center $s$ to refurbishing center $r$

$s v_{r p}$ : satisfaction of fast delivery of parts from refurbishing center $r$ to part inventory $p$

$s n_{p m}$ : satisfaction of fast delivery of parts from part inventory $p$ to producer $m$

$s g_{k m}$ : satisfaction of fast delivery of parts from supplier $k$ to producer $m$

$\mathrm{fm}_{m}$ : cost of locating a producer in suggested location $m$

$f d_{d}$ : cost of locating a distributor in suggested location $d$

$f s_{s}$ : cost of locating a disassembly center in suggested location $s$

$f r_{r}$ : cost of locating a refurbishing center in suggested location $r$

$f p_{p}$ : cost of locating a part inventory in suggested location $p$

$o m_{j m}$ : operational cost for a product $j$ in producer $m$

$o d_{j d}$ : operational cost for a product $j$ in distributor $d$

$o c_{j c}$ : operational cost for a product $j$ in retailer $c$

$o s_{i s}$ : operational cost for a part $i$ in disassembly center $s$

$o r_{i r}$ : operational cost for a part $i$ in refurbishing center $r$

$o p_{i p}$ : operational cost for a part $i$ in part inventory $p$

$q m_{m}$ : cost of producer $m$ 's opportunity cost

$q d_{d}$ : cost of distributor $d$ 's opportunity cost

$q s_{s}$ : cost of disassembly center $d$ 's opportunity cost

$q r_{r}$ : cost of refurbishing center $r$ 's opportunity cost

$q p_{p}:$ cost of part inventory $p$ 's opportunity cost

$e m_{m}$ : pollution amount of capacity surplus in producer $m$

$e d_{d}$ : pollution amount of capacity surplus in distributor $d$

$e s_{s}$ : pollution amount of capacity surplus in disassembly center $s$

$e r_{r}$ : pollution amount of capacity surplus in refurbishing center $r$

$e p_{p}$ : pollution amount of capacity surplus in part inventory $p$

$p m_{m}$ : pollution amount of producer $m$ 
$p d_{d}$ : pollution amount of distributor $d$

$p s_{s}$ : pollution amount of disassembly center $s$

$p r_{r}$ : pollution amount of refurbishing center $r$

$p p_{p}$ : pollution amount of part inventory $p$

$d m$ : satisfaction of dispersion and variety of producer's facilities

$d d:$ satisfaction of dispersion and variety of distributor's facilities

$d s:$ satisfaction of dispersion and variety of disassembly center's facilities

$d r$ : satisfaction of dispersion and variety of refurbishing center's facilities

$d p$ : satisfaction of dispersion and variety of part inventory's facilities

$s m_{m}$ : satisfaction from effects of capacity surplus of producer $m$

$s d_{d}$ : satisfaction from effects of capacity surplus of distributor $d$

$s s_{s}$ : satisfaction from effects of capacity surplus of disassembly center $s$

$s r_{r}$ : satisfaction from effects of capacity surplus of refurbishing center $r$

$s p_{p}$ : satisfaction from effects of capacity surplus of part inventory $p$

$c p_{p}:$ maximum capacity of part inventory $p$

$c d_{d}$ : maximum capacity of distributor $d$

$c r_{r}$ : maximum capacity of refurbishing center $r$

$\mathrm{cm}_{m}$ : maximum capacity of producer $m$

$c s_{s}$ : maximum capacity of disassembly center $s$

$s p r_{i k}$ : buying price of part $i$ from supplier $k$

$q_{i j}:$ number of part $i$ in product $j$

$y_{j}:$ return percentage of product $j$

$w_{i}$ : uselessness of part $i$

$s_{j c}:$ price of product $j$ in retail $c$

$v s_{j c}$ : salvage value of unsold product $j$ in retail $c$

$u s_{j c}$ : shortage cost of lack of product $j$ in retail $c$

$D_{j c}$ : demand for product $j$ in retail $c$

$E\left(D_{j c)}\right.$ : expected demand $j$ in retail $c$

$F\left(D_{j c)}\right.$ : cumulative distribution function of variable $D_{j c}$

$M$ : big positive number 
Decision variables

$g_{i k m t}$ : the amount of part $i$ which will be sent by transportation vehicles $t$ from supplier $k$ to producer $m$

$f_{j m d t}$ : the amount of product $j$ which will be sent by transportation vehicles $t$ from producer $m$ to distributor $d$

$e_{j d c t}$ : the amount of product $j$ which will be sent by transportation vehicles $t$ from distributor $d$ to retailer $c$

$h_{j c s t}$ : the amount of product $j$ which will be sent by transportation vehicles $t$ from retailer $c$ to disassembly center $s$

$l_{\text {isrt }}$ : the amount of part $i$ which will be sent by transportation vehicles $t$ from disassembly center $s$ to refurbishing center $r$

$v_{\text {irpt }}$ : the amount of part $i$ which will be sent by transportation vehicles $t$ from refurbishing center $r$ to part inventory $p$

$n_{\text {ipmt }}$ : the amount of part $i$ which will be sent by transportation vehicles $t$ from part inventory $p$ to producer $m$

$x m_{m}$ : equals 1 if a producer located in suggested place $\mathrm{m}$ and equals to 0 otherwise

$x d_{d}$ : equals 1 if a distributor located in suggested place $\mathrm{d}$ and equals to 0 otherwise

$x s_{s}$ : equals 1 if a disassembly center located in suggested place s and equals to 0 otherwise

$x r_{r}$ : equals 1 if a refurbishing center located in suggested place $\mathrm{r}$ and equals to 0 otherwise

$x p_{p}$ : equals 1 if a part inventory located in suggested place $\mathrm{p}$ and equals to 0 otherwise

$a m_{m}$ : capacity percentage of producer $m$ which is used. Between 0 and 1

$a d_{d}$ : capacity percentage of distributor $d$ which is used. Between 0 and 1

$a s_{s}$ : capacity percentage of disassembly center $s$ which is used. Between 0 and 1

$a r_{r}$ : capacity percentage of refurbishing center $r$ which is used. Between 0 and 1

$a p_{p}$ : capacity percentage of part inventory $p$ which is used. Between 0 and 1

$o_{j m}$ : amount of product $j$ produced in producer $m$

$b_{i s}$ : amount of part $i$ disassembled in disassembly center $s$

$x_{j c}$ : amount of product $j$ sent to retailer $c$

Parameters and variables of the network showed schematically in Figure 1. 


\section{Appendix B}

Simplification of profit formula of products in retailers As mentioned in formula (1), profit of product $\mathrm{j}$ in retailer $\mathrm{c}$ is as follows:

$$
s_{j c}\left(x_{j c} \wedge D_{j c}\right)+s v_{j c}\left(x_{j c}-D_{j c}\right)^{+}-s c_{j c}\left(D_{j c}-x_{j c}\right)^{+}
$$

Which can be simplified using the following equations:

$$
\begin{aligned}
& =s_{j c}\left(x_{j c} \wedge D_{j c}\right)+s v_{j c}\left(x_{j c}-D_{j c}\right)^{+}-s c_{j c}\left(D_{j c}-x_{j c}\right)^{+} \\
& =s_{j c}\left(x_{j c}-\left(x_{j c}-D_{j c}\right)^{+}\right)+s v_{j c}\left(x_{j c}-D_{j c}\right)^{+}-s c_{j c}\left(D_{j c}-x_{j c}\right)^{+} \\
& =s_{j c} x_{j c}+\left(x_{j c}-D_{j c}\right)^{+}\left(-s_{j c}+s v_{j c}\right)+\left(D_{j c}-x_{j c}\right)^{+}\left(-s c_{j c}\right) \\
& =s_{j c} x_{j c}+\left(x_{j c}-D_{j c}\right)^{+}\left(-s_{j c}+s v_{j c}\right)+\left(\left(x_{j c}-D_{j c}\right)^{+}-\left(x_{j c}-E\left(D_{j c}\right)\right)\right)\left(-s c_{j c}\right) \\
& =\left(s_{j c}+s c_{j c}\right) x_{j c}+\left(x_{j c}-D_{j c}\right)^{+}\left(s v_{j c}-s_{j c}-s c_{j c}\right)-E\left(D_{j c}\right) s c_{j c} \\
& =\left(s_{j c}+s c_{j c}\right) x_{j c}+\left(s v_{j c}-s_{j c}-s c_{j c}\right) \int_{0}^{x_{j c}} F\left(D_{j c}\right) d D_{j c}-E\left(D_{j c}\right) u s_{j c}
\end{aligned}
$$

